\title{
Simultaneous Complex Open Dorsal Dislocation of Four Metacarpophalangeal Joints
}

\author{
Mehran Razavipour, ${ }^{1}$ Masoud Shayesteh Azar, ${ }^{1}$ Mohammad Hossein Kariminasab, and \\ Seyed Mohamad Mehdi Daneshpoor ${ }^{1, *}$ \\ ${ }^{1}$ Orthopedic Research Center, Mazandaran University of Medical Sciences, Sari, IR Iran \\ *Corresponding author: Seyed Mohamad Mehdi Daneshpoor, Orthopedic Research Center, Mazandaran University of Medical Sciences, Sari, IR Iran. Tel: +98-1133361700, Fax: \\ +98-1133361703, E-mail: mdaneshpour@mazums.ac.ir
}

Received 2015 June 23; Revised 2015 August 25; Accepted 2015 September 22.

\begin{abstract}
Introduction: Metacarpophalangeal (MCP) joints dislocations are rare, especially the simultaneous complex dislocations of four MCP joints. Although there are over 100 reported cases of MCP joint dislocation, the vast majority involves the index finger and is extremely rare in the long or ring fingers. Up to present, only three cases of simultaneous complex dorsal dislocation of four joints were described in the literature.

Case Presentation: We present a rare case of complex dorsal dislocation of four MCP joints. Physical examination and radiographic evaluation revealed open dorsal MCP joint dislocation of index, middle, ring and little fingers. Any attempt of closed reduction was unsuccessful, before surgery. The patient in the case underwent open reduction and, after surgery, the injured hand was placed in a short extension block splint and controlled active motion, while in splint, was encouraged, immediately. This approach resulted in an uneventful recovery.

Conclusions: We described the complex dorsal MCP joint dislocation of four joints, which is defined as irreducible and complete dislocation, most often because of volar plate interposition. Although the dorsal and volar approaches are common methods of treatment, in complex MCP joint dislocations, however, to our knowledge, the dorsal approach has better advantages, compared with the volar type.
\end{abstract}

Keywords: Dislocations, Metacarpophalangeal Joint, Volar Plate

\section{Introduction}

The metacarpophalageal (MCP) joints are resistant to trauma because of tight capsuloligamentous structures. Therefore, these dislocations are rare, especially the simultaneous complex dislocations of four MCP joints ( 1 , 2 ). It has been first described by McCarthy, in 1980, and classified, based on direction of translation, as volar or dorsal and, also, incomplete, simple complete and complex complete dislocation (3-5).

Complex dorsal MCP joint dislocation is defined as irreducible and complete dislocation most often because of volar plate interposition. It occurs most frequently in the index finger and is extremely rare in the long or ring fingers. It should be notified that longitudinal traction may convert a simple dislocation into a complex dislocation (6). The dislocation should be reduced within several hours.

We present a rare case of complex dorsal dislocation of four MCP joints that was treated with open reduction and an extension block splint.

\section{Case Presentation}

We report a 30-year-old Iranian, right hand dominant, male worker, who fell on outstretched hand, from the third floor, during work, and injured his left hand. He suffered from severe pain in his left hand. Claw-like position of the hand was significant, upon presentation (Figure 1) and a transverse laceration, of approximately $5 \mathrm{~cm}$ in length, was detected in the distal palm of his left hand. On physical examination, the patient was unable to flex his fingers. Deformity of the MCP joints was prominent at presentation and, also, the metacarpal heads were visible at the bottom of the wound (Figure 2). Two point discrimination and capillary refill were normal. Radiographs confirmed dorsal dislocation of four MCP joints and there were no evidences of associated fractures (Figure 3 ).

The patient underwent surgery and the laceration was vigorously irrigated with saline solution. A gentle attempt to closed reduction of the MCP joints was unsuccessful. Consequently, open reduction was performed, via dorsal approach, with two longitudinal incisions. The metacarpal heads were exposed and palpated, at the bottom of the laceration. The MCP joints were stable, after open reduction in arc of 0 - 90 degrees. The wound was repaired with 4 - 0 nylon sutures and intravenous antibiotics were administered, for 48 hours. Active range of

Copyright (C) 2015, Iran University of Medical Sciences. This is an open-access article distributed under the terms of the Creative Commons Attribution-NonCommercial 4.0 International License (http://creativecommons.org/licenses/by-nc/4.0/) which permits copy and redistribute the material just in noncommercial usages, provided the original work is properly cited. 


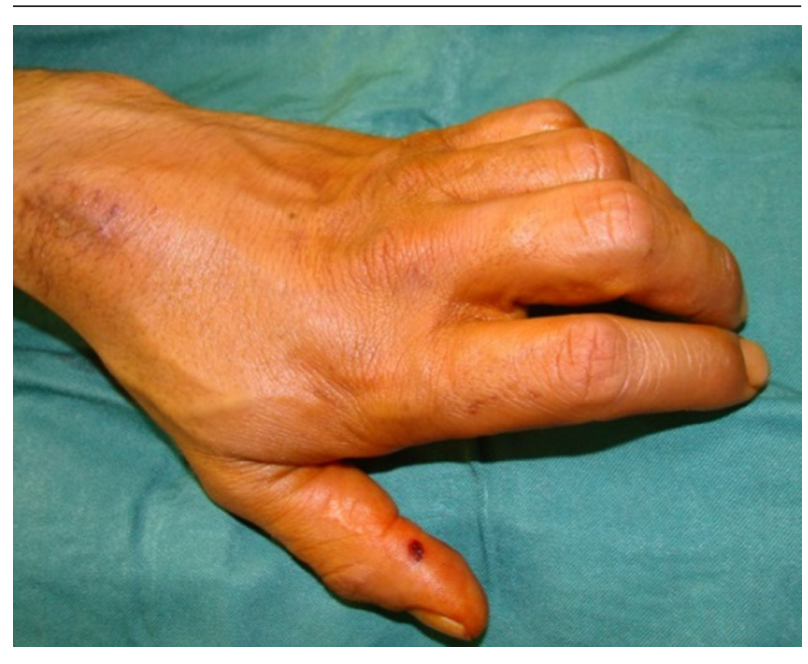

Figure 1. Claw-Like Position of Patient's Hand Upon Presentation

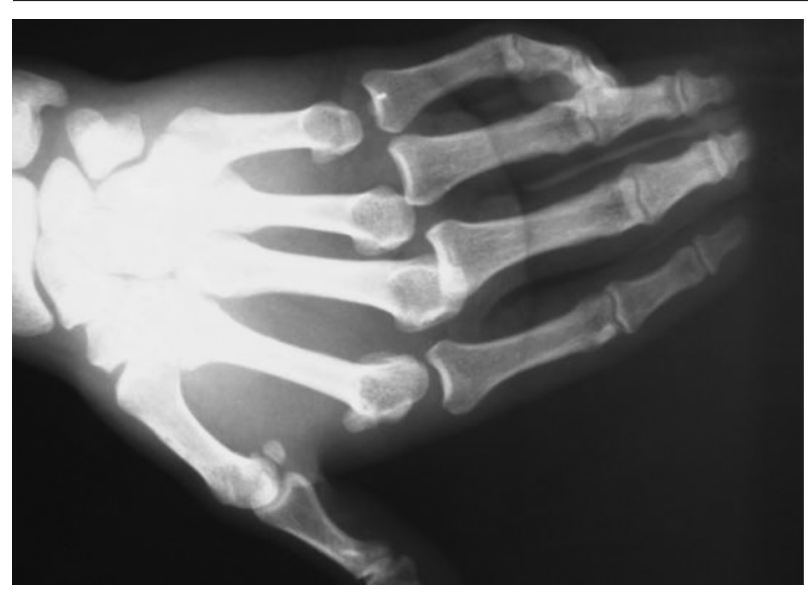

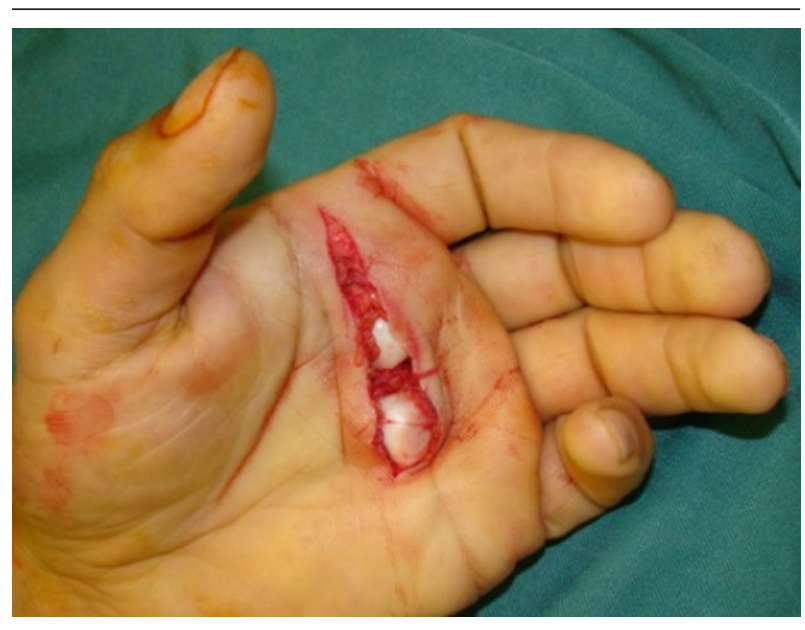

Figure 2. Transverse Laceration on the Palmar Surface of Injured Hand, With Exposure of Metacarpal Heads

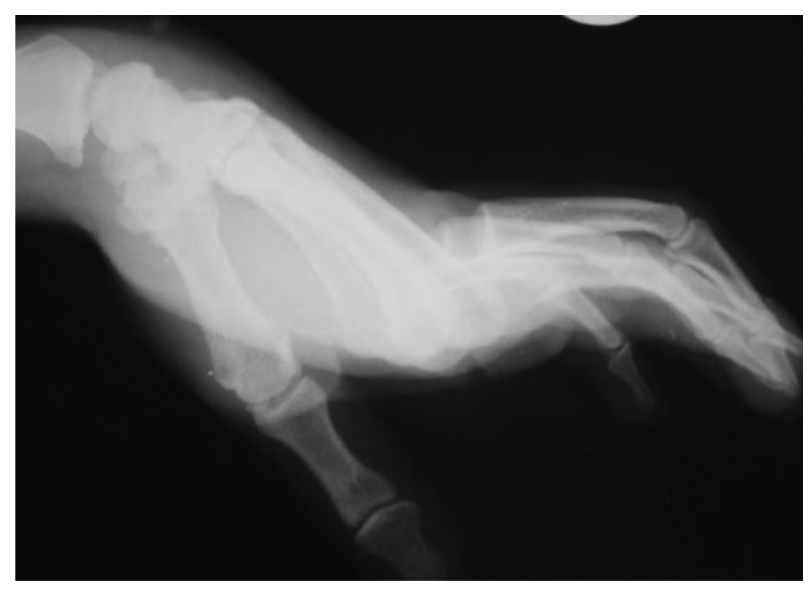

Figure 3. Anteroposterior and Lateral X-Ray Evidencing Dorsal Dislocation of Four Metacarpophalangeal Joints Without Associated Fractures

motion, without limitation, was started after splint removal, 3 weeks later. Then, we prescribed physiotherapy by a trained physiotherapist, for 10 sessions, in a daily routine.

Hand functionality was restored completely, without any complication. After discharge, the patient was followed for 6 months and no complication occurred.

\section{Discussion}

The MCP joints dislocations were first described by Polaillon in 1884 (4). They are infrequent and the simultaneous complex dislocations of four MCP joints are extremely rare, the first being described by McCarthy in 1980 (1). Kaplan revealed rupture of the volar plate, from its proximal attachment to the metacarpal bone, for the first time in complex MCP joint dislocations (3).

The anatomy of the MCP joint is responsible for inherent stability and infrequent dislocation. Radial and ulnar collateral ligaments play primary roles in the stability of this joint (5). These dislocations usually occur as a result of falling on an outstretched hand $(1,3)$ and can be classified, based on the direction of translation, as volar or dorsal and, also, incomplete, simple complete and complex complete dislocations, based on the volar plate entrapment (5).

The longer is reduction delayed, the more important are the degenerative changes and less satisfactory results, in the pain score and joint stability, will be seen $(7,8)$. If a MCP dislocation is not reduced carefully, a simple MCP joint dislocation can be converted into a complex, irreducible type and become a time consuming procedure (9).

On radiographic assessment, a visible sesamoid bone, in the joint space, is pathognomonic of complex dislocation. This subject indicates that the volar plate has become entrapped in the MCP joint (10).

Even in complex dislocations, one gentle attempt of closed reduction, under general anesthesia and muscle relaxation, should be considered. There are several problems, such as volar plate entrapment in the joint and dorsal displaced flexor tendons that cause failure 
of reduction. In such circumstance, we use the open reduction approach for the dislocation, although other authors, like Stowell et al. believed that reduction should be closed when the dislocation is simple and open reduction should be performed for complex dislocations, in which the volar plate become entrapped in the joint (9).

An important point in the open reduction technique is to assess the osteochondral fracture and remove all anatomical structures, which are entrapped in the joint, including the volar plate $(5,10)$. Despite this subject, Stowell et al. (9) suggested that closed reduction may be definitive treatment. They proposed that closed reduction may be sufficient, if there is only a short time delay between the dislocation and treatment procedure. Therefore, soft tissue repair may not be necessary in all cases. On the other hand, they suggested that gentle tissue manipulation, with copious irrigation and appropriate antibiotic coverage, can result in a patient outcome that is equal to open reduction, without the risk of digital nerve damage, during joint exposure (9).

There was a certain amount of debate regarding the selection of the surgical approach for this dislocation, in the past, because Kaplan (3), Baltas (11) and Durakbasa and Guneri (4) believed that volar approach is a better choice for complex MCP joint dislocations. They used the volar approach because this could show anatomic structures more accurately $(3,4,11)$, whereas Izadpanah and Wanzel (5) and Patterson et al. (7) agreed on a dorsal approach. This approach has first been described by Farabeuf, in 1876 (7), which showed the disadvantages of volar approach, such as digital nerve injury. Furthermore, they revealed that the dorsal approach has numerous advantages, such as decreased probability of digital nerve injury, more accurate management of the associated osteochondral fractures, and a better visualization of the volar plate that was entrapped $(5,7)$. Based on these advantages, we used the dorsal approach with two longitudinal incisions, for the open reduction of this patient.

To be noted, the determination of MCP joint stability should be tested at the end of the operation that shows the collateral ligament status. Other controversy regards the immobilization period, after the reduction. Several authors mitigate for early mobilization (8), whereas others suggest immobilization after the procedure. When the joint is stable, the use of an extension block splint will be enough. A minimum time of immobilization ( 2 or 3 weeks) is advisable and active motion should be started after splint removal, for prevention of joint stiffness $(4,5,7)$.

In conclusion, in these dislocations, immediate surgical intervention, such as irrigation, debridement and reduction, should be done. It should be kept in mind that a visible sesamoid bone in the joint space, on radiographs, is pathognomonic for complex dislocation. Despite of this, at first, one attempt of closed reduction should be considered (10). To our knowledge, this case is the fourth to be described until now, which makes our particular experience extremely rare. As a final result, we reveal that, although the dorsal and volar approaches are common methods of treatment in complex MCP joint dislocations, to our knowledge, the dorsal approach has better advantages, compared with the volar type.

\section{References}

1. McCarthy LJ. Open metacarpophalangeal dislocations of the index, middle, ring, and little fingers. J Trauma. 1980;20(2):183-5. [PubMed: 7354503]

2. Wright CS. Compound dislocations of four metacarpophalangeal joints. J Hand Surg Br. 1985;10(2):233-5. [PubMed: 4031611]

3. Kaplan EB. Dorsal dislocation of the metacarpophalangeal joint of the index finger. J Bone Joint Surg Am. 1957;39-A(5):1081-6. [PubMed: 13475407]

4. Durakbasa O, Guneri B. The volar surgical approach in complex dorsal metacarpophalangeal dislocations. Injury. 2009;40(6):657-9. doi: 10.1016/j.injury.2008.10.027. [PubMed: 19232591]

5. Izadpanah A, Wanzel K. Late presentation of a complete complex thumb metacarpophalangeal joint dislocation: A case report. Can J Plast Surg. 2011;19(4):139-42. [PubMed: 23204885]

6. Minami A, An KN, Cooney WP, Linscheid RL, Chao EY. Ligament stability of the metacarpophalangeal joint: a biomechanical study.J Hand Surg Am. 1985;10(2):255-60. [PubMed:3980940]

7. Patterson RW, Maschke SD, Evans PJ, Lawton JN. Dorsal approach for open reduction of complex metacarpophalangeal joint dislocations. Orthopedics. 2008;31(11):1099. [PubMed:19226090]

8. Eglseder WA, Gens DR, Burgess AR. Multiple ipsilateral dorsal metacarpophalangeal and proximal interphalangeal joint dislocations: a case report. J Trauma. 1995;38(6):955-7. [PubMed: 7602645]

9. Stowell JF, Rennie WP. Simultaneous open and closed dislocations of adjacent metacarpophalangeal joints: a case report. $J$ Emerg Med. 2002;23(4):355-8. [PubMed:12480014]

10. Silberman WW. Clear view of the index sesamoid: a sign of irreducible metacarpophalangeal joint dislocation. JACEP. 1979;8(9):371-3. [PubMed: 470281]

11. Baltas D. Complex dislocation of the metacarpophalangeal joint of the index finger with sesamoid entrapment. Injury. 1995;26(2):123-5. [PubMed: 7721465] 